\title{
Development of Pulse Magnetization System on Aberration Corrected 1.2-MV Cold Field-Emission Transmission Electron Microscope
}

Toshiaki Tanigaki ${ }^{1}$, Tetsuya Akashi ${ }^{1}$, Akira Sugawara ${ }^{1}$, Kodai Niitsu ${ }^{2}$, Xiuzhen $\mathrm{Yu}^{2}$, Yasuhide Tomioka $^{3}$, Daisuke Shindo ${ }^{2,4}$, Yoshinori Tokura ${ }^{2,5}$ and Hiroyuki Shinada ${ }^{1}$

1. Research \& Development Group, Hitachi, Ltd., Hatoyama 350-0395, Japan

2. Center for Emergent Matter Science (CEMS), RIKEN, Wako 351-0198, Japan

3. National Institute of Advanced Industrial Science and Technology (AIST), Tsukuba 305-8568, Japan

4. Institute of Multidisciplinary Research for Advanced Materials, Tohoku University, Sendai 980-8577, Japan

5. Department of Applied Physics and Quantum-Phase Electron Center (QPEC), University of Tokyo, Tokyo 113-8656, Japan

Observations of structures and electromagnetic fields at atomic resolution provide critical information for material science and industrial research. A transmission electron microscope (TEM) has advantage in high-resolution observation with capability of seeing inside the sample. In the early days, the spatial resolution of the electron microscope has been improved by increasing accelerating voltages. After realization of aberration corrector, the atomic arrangements became observable using wide range of accelerating voltages. For observing electromagnetic filed at atomic scale using electron holography, we developed 1.2-MV TEM equipped with a cold field-emission gun and a CEOS hexapole spherical aberration corrector. The spatial resolution has reached $0.043 \mathrm{~nm}$ at the high-resolution observation conditions, in which the sample is placed in high magnetic field of the objective lens [1]. Under the observation condition, in which the sample is placed in a field-free position for observing magnetic field, the special resolution has been reached $0.21 \mathrm{~nm} \mathrm{[2].} \mathrm{The} \mathrm{observation} \mathrm{of} \mathrm{magnetic} \mathrm{field} \mathrm{at} \mathrm{atomic}$ resolution using electron holography is expected to be realized, however, the phase resolution of magnetic phase measurement has to be increased around $2 \pi / 1000 \mathrm{rad}$ for this goal.

The phase resolution of electron holography has been reached $2 \pi / 1000 \mathrm{rad}$ in vacuum area [3]. However, the phase resolution of magnetic phase measurement in the sample area has been around $2 \pi / 200 \mathrm{rad}$ [4] because of difficulties in data acquisition to separate electrostatic and magnetic contributions in the phase shift of the electron waves. Here we report the development of pulse magnetization system on 1.2MV TEM for increasing the phase resolution by changing only magnetization direction between two acquisition conditions of electron holograms.

Magnetization direction in the sample is reversed by pulse magnetic fields generated by coils placed near both sides of sample holder. The system has been designed to be used with double tilt sample holder or other holders for realizing varieties of observation conditions. The maximum magnetic field at sample position applied by the system was $5200 \mathrm{G}$.

The magnetization reversal in $\mathrm{Ba}_{2} \mathrm{FeMoO}_{6}$ thin sample by applying $2600 \mathrm{G}$ was confirmed by electron holography (Figure 1). The central area of the sample was observed at higher magnification by electron holography and magnetization reversals were repeated 140 times for reducing statistical noise in the phase measurements. The magnetic phase image and its profiles are shown in Figure 2. The phase noise in the magnetic phase measurements was evaluated by assuming constant magnetization, which gives linear phase profile. The obtained phase noise of $2 \pi / 2990 \mathrm{rad}$ corresponds to the phase resolution of 
$2 \pi / 997 \mathrm{rad}$ in the case of setting signal to noise ratio to be 3 .

The developed pulse magnetization system on 1.2-MV TEM can be used for increasing the magnetic phase resolution in electron holography. The magnetic phase resolution obtained by using the system has been reached $2 \pi / 1000 \mathrm{rad}$ in the sample area. The pulse magnetization system is thought to be promising technologies for realizing magnetic field observations at atomic scale.

\section{References:}

[1] T Akashi et al, Appl. Phys. Lett. 106 (2015), p.074101.

[2] Y. Takahashi et al., Microsc. Microanal. 21(Suppl 3) (2015), p.1865.

[3] T. Suzuki et al., Ultramicroscopy 118 (2012) p.21.

[4] Y. Murakami et al., Nature Commnications 5 (2014) p.4133.

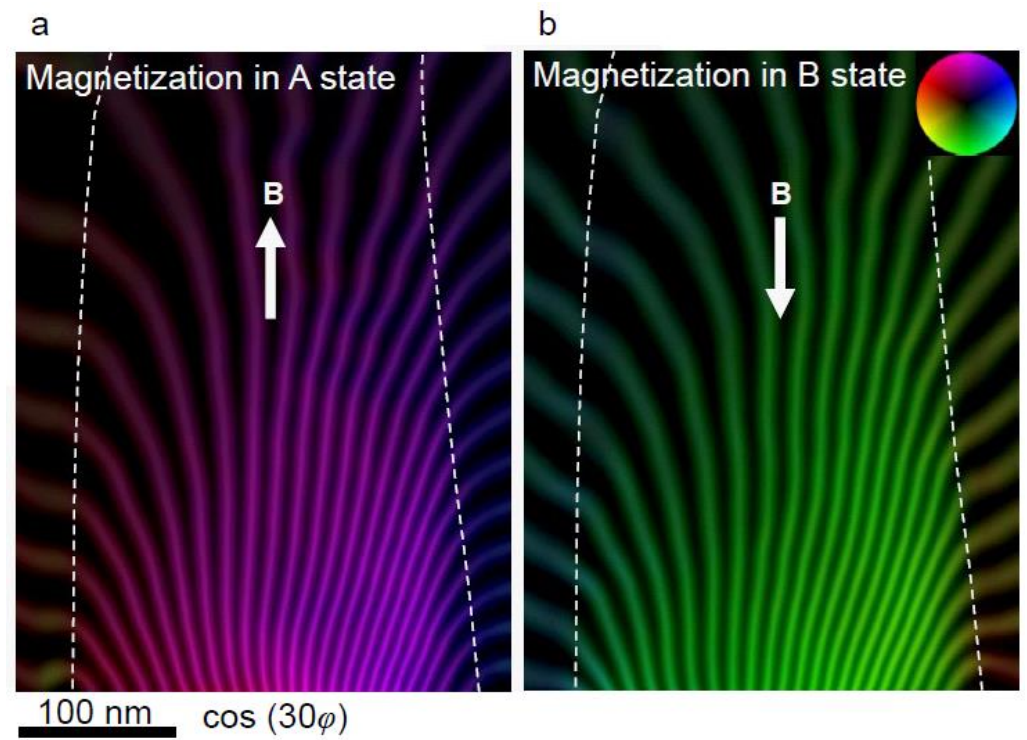

Figure 1. Magnetization reversal using developed pulse magnetization was confirmed by electron holography. White dashed lines indicate the edges of thin sample. Magnetization in A state (a) is opposite to that in B state (b).

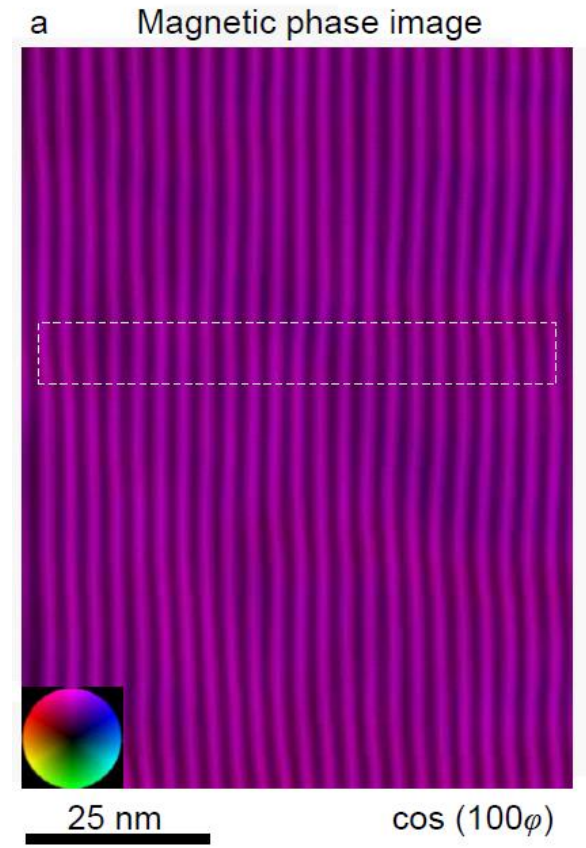

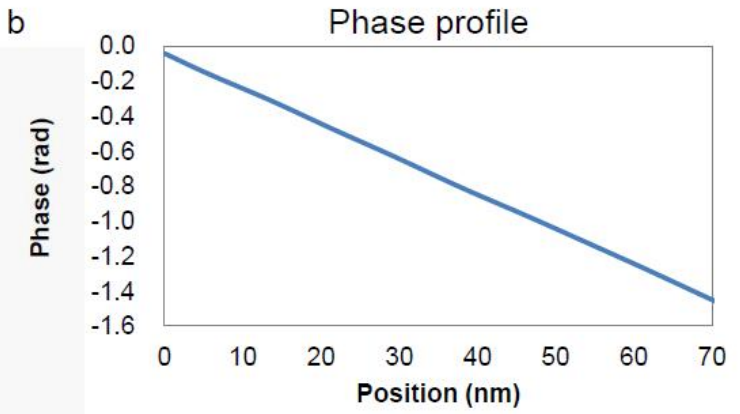

$\circ$

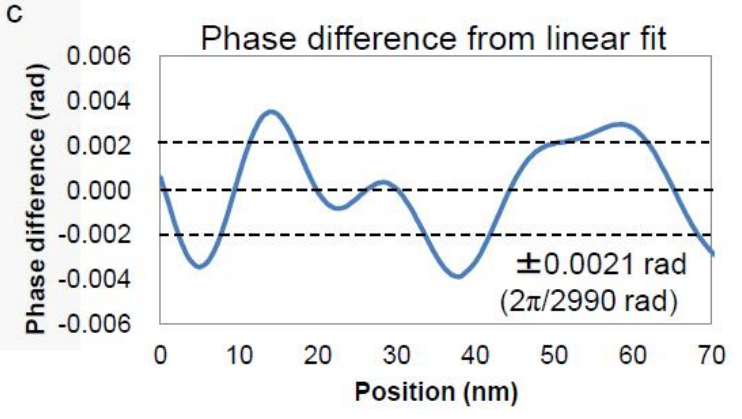

Figure 2. Magnetic phase at central area of the sample obtained by using the pulse magnetization system. (a) Magnetic phase image. (b) Phase profile. (c) Phase difference from linear fit. 